\title{
Testing Generative Models of Online Collaboration with BigBang
}

\author{
Sebastian Benthall ${ }^{\text {** }}$ \\ https://www.youtube.com/watch?v=AQFS_ES7rT0
}

\begin{abstract}
We introduce BigBang, a new Python toolkit for analyzing online collaborative communities such as those that build open source software. Mailing lists serve as critical communications infrastructure for many communities, including several of the open source software development communities that build scientific Python packages. BigBang provides tools for analyzing mailing lists. As a demonstration, in this paper we test a generative model of network growth on collaborative communities. We derive social networks from archival mailing list history and test the Barabási-Alpert model against this data. We find the model does not fit the data, but that mailing list social networks share statistical regularities. This suggests room for a new generative model of network formation in the open collaborative setting.
\end{abstract}

Index Terms - mailing lists, network analysis, assortativity, power law distributions, collaboration

\section{Introduction}

Open source software communites such as those that produce many scientific Python packages are a critical part of contemporary scientific organization. A distinguishing feature of these communities is their heavy use of Internet-based infrastructure, such as mailing lists, version control systems, and issue trackers, for managing communications and organizing work on distributed teams. This data is often deliberately publicly accessible as open source best practices include the "conspicuous use of archives" [Fogel]. The availability of these digital records are also an excellent resource for the researcher interested in sociotechnical organization and collaboration within science.

This paper introduces BigBang, a Python project whose purpose is the collection, preprocessing, and analysis of data from open collaborative communities. Built for the use case of studying the Scientific Python communities in particular, it generalizes to other communities and supports fruitful comparisons between them.

To demonstrate the potential of this approach, this paper will explore the structure of mailing list discussions in the context of open collaborative projects. We extract social network data from the archives of public mailing lists and test the plausibility that these graphs were generated by Barabási-Alpert network model. We find that of the mailing lists we've analyzed, none exhibit

* Corresponding author: sb@ischool.berkeley.edu

¥UC Berkeley School of Information

Copyright $(2015$ Sebastian Benthall. This is an open-access article distributed under the terms of the Creative Commons Attribution License, which permits unrestricted use, distribution, and reproduction in any medium, provided the original author and source are credited. two features of Barabási-Alpert networks: power law degree distribution and zero degree assortativity. Instead the data indicates that these networks have a log-normal degree distribution and have negative degree disassortivity. This result suggests the possibility of future work of scientifically developing a generative model of collaboration.

\section{BigBang Overview}

Launched in 2014, BigBang is a software project that aims to provide researchers a complete toolkit for the scientific analysis of open online collaborative communities. Though applicable to many domains, research into online collaboration has special relevance to practicioners of computationally intensive open science. Through it scientific programming communities such as Scientific Python can achieve a quantitative understanding of their own work and innovation process.

Thorough study of these kinds of communities requires the collection and rationalization of many heterogenous and highdimensional data sources, including but not limited to mailing lists, version control systems such as Git, and issue trackers such as GitHub and Bugzilla.

This data is complex in that it has many dimensions that afford very different kinds of analysis:

- Time. All data from online collaboration infrastructure is timestamped, affording use of time series methods.

- Text. Email message bodies, issue contents, and commit messages in version control are all text data suitable for study with natural language processing techniques.

- Social network. Participants in the project are individuals linked by relational ties of communication. Hence these data afford study through social network analysis techniques.

- Software static analysis. Source code in version control is complex data containing the definition of many interelated variables, functions, classes, and modules. Static analysis and compilation techniques from computer science can be used to study these entities within the software itself.

The richness and granularity of the data from open source software communities and other open on-line collaborative projects promise the answers to many research questions about software engineering, innovation, social organization, and more. The catch is that with data that is so multifaceted, preprocessing the data is an engineering-intensive endeavor. 
The versatility and scope of open source Scientific Python packages makes building a generic research infrastructure for analyzing these communities a possibility within reach. Architecturally, BigBang is a Python package that includes Scientific Python libraries for time series analysis, natural language processing, network analysis, and software analysis as dependencies.

BigBang includes methods for collecting research data from sources on the web about the activity of open collaborative communities. At the time of this writing, BigBang supports data collection from Mailman, the mailing list service, as well as other .mbox formatted email archives. It also supports data collection from Git repositories. Future versions will have methods for collecting data from issue trackers.

The BigBang repository contains an examples directory of Jupyter notebooks demonstrating its functionality and exploring lines of research inquiry. Researchers can contribute to the project by submitting Jupyter notebooks to the repository for review through the GitHub Pull Request system. Source code that performs preprocessing that is usable by multiple computational experiments is in a separate source code directory that can be imported as a Python module.

In the context of Scientific Python, BigBang is deliberately recursive. It is a Python project that depends on many other scientific Python projects. It is designed to study, among other things, dependencies and interactions between the Scientific Python technologies and communities. Our goal is for BigBang to provide a new means for these communities to engage in scientific selfmanagement.

\section{Testing Generative Models of Online Collaboration}

As a demonstration of BigBang's capabilities, in this paper we will test a well known generative model of network formation against social network data derived from public mailing list discussions. A generative model is a formal model that describes a process through which data is generated. A principle benefit of a formal generative model is that the statistical properties of data it generates can be compared with the statistical properties of empirical data. Such comparisons are one way to get empirical purchase on the mechanism behind even purely observational data. Discovering a concise generative model that fits data from online collaboration would give us insight into the mechanism of collaboration itself.

In this paper, we will test one well known generative model of network data, the Barabási-Alpert model. This model describes a process by which new nodes, as they join a network, form edges with other nodes with probability proportional to their degree. This process is called preferential attachment. Very roughly speaking, in social networks preferential attachment is suggestive of a network dominated by attachments to a small number of luminaries. In its basic form, this model generates networks with two notable statistical properties:

- The degree distributions of Barabási-Alpert networks are scale-free, meaning that the fraction of nodes of degree $k$ falls asymptotically according to a power law distribution. $P(k) \sim k^{-\gamma}$ for some positive $\gamma$.

- The correlation between the degrees of adjacent nodes converges to zero (from below) as the network grows.

We discover in our empirical data that neither of these properties hold for the social networks fo public mailing list discussions.
This suggests that preferential attachment is not a mechanism that dominates the social interactions on the collaborative projects represented in our data. On the contrary, the statistical properties of public mailing list discussions suggest that participation is more widely distributed than in many other social networks, and that interaction with new participations is a priority.

\section{Preferential attachment model}

An early result in the study of complex networks was the observation that many networks existing in nature exhibit a scale-free degree distribution. [BarabásiAlbert] This means that the tail of the distribution of the number of edges of each node in the network (the node's degree) converges to a power law function:

$$
y=a x^{k}
$$

(Scale-free refers to the scale invariance of the power law distribution.)

The prevalence of scale-free networks in nature has raised the question of what generative processes produce networks with this property. What was at one point the most well-known random graph model, the Erdős-Rénri model, produces networks with binomial degree distribution. Barabási and Alpert [BarabásiAlbert] have proposed a widely cited and studied model of network generation that produces graphs with scale-free degree distribution.

The attractiveness of the Barabási-Alpert model is due in part to its being a generative model that describes a process for creating data of an observed distribution, as opposed to being simply a description of the distribution itself. This gives the Barabási-Alpert model explanatory power.

In particular, the Barabási-Alpert model attributes the scalefree distribution of node degree to a preferential attachment mechanism, parameterized by $m_{0}$ and $m$. The network is formed by beginning with a small number $m_{0}$ of nodes and adding new nodes, connecting each new node to $m<m_{0}$ nodes, where the probability of connecting to node $i$ is proportional to the prior degree of that node, $k_{i}$.

$$
P\left(k_{i}\right)=\frac{k_{i}}{\sum_{j} k_{j}}
$$

Here, $\sum_{j} k_{j}$ is the sum of all degrees of all nodes in the graph. The parameter $m$ is fixed across all iterations. [AlbertBarabási]

The Barabási-Alpert model is favored for its simplicity, its intuitively clear mechanism of preferential attachment, and for its analytic tractability. Intuitively, a social process driven by preferential attachment is one in which "the rich get richer". Consider the social graph from an on-line social network such as Facebook or Twitter. New entrants to the network will 'friend' or 'follow' existing nodes. If they preferentially attach, the will be much more likely to connect to celebrities who already dominate the network than to new entrants such as themselves. The most highly connected participants will likely owe their position in the network to their seniority. Studies have supported the role of a preferential attachment mechanism in social network formation [Zhou2011], [Tinatti2012]. It is an empirical question whether the preferential attachment mechanism explains the data from collaborative communities such as those that develop Scientific Python packages. 


\section{Power law or log-normal?}

An implicit challenge to the Barabási-Alpert model comes from [Clauset2007], who argue that many conventionally accepted techniques for fitting power law distributions to empirical data are biased and unsound. Specifically, they critique the common method of plotting the histogram of the data on a log-log axis and testing for linearity by perfoming a least-squares linear regression, and variations of this. They propose an alternative Bayesian technique for testing power law distributions. By computing the likelihood of the data being generated by a power law distribution and comparing it with the likelihood of it being generated by other heavy-tail distributions, such as the log-normal distribution, they provide a statistically sound basis for model comparison.

The Clauset et al. method considers only the tail of the data, picking a cutoff value $x_{\min }$ below which data are ignored. They argue that picking this value is of critical importance: to pick too high an $x_{\min }$ is to fit a power law to non-power law data. to pick too low a value is to throw out legitimate data, which can lead to bias. They propose selecting the $x_{\text {min }}$ that minimizes the KolmogorovSmirnov distance between the best fitting power law distribution and the empirical data above the mininum.

We refer the reader to [Clauset2007] for the in-depth defense of this method as an alternative to those based on testing for linearity on a log-log scale. Besides its statistical soundess, an advantage of this method is that it has been implemented in Python in the powerlaw package by [Alstott2014], which is what is used for the computational results below.

From a Bayesian perspective, the ratio of likelihoods represents how much one should update one's beliefs based on observation of data. In this case, the computed likelihood ratio of the data being generated by a power law over a log-normal distribution would be interpreted as how much the data should persuade that it came from a power law distribution independent of one's prior untested belief.

This leaves open the question of the prior probability of a distribution being generated by a power law producing process, or a log-normal producing process. [Mitzenmacher2003] surveys a century of scientific disagreement over the prevalance of each distribution across many disciplines. Different processes are expected to produce different distributions.

Processes through which "the rich get richer" systematically, such as the preferential attachment process described above, will produce power law distributed data.

Log-normal distributions are produced by what [Mitzenmacher2003] calls multiplicative processes. A multiplicative process occurs when independent random variables are multiplied together. Contrast this with the preferential attachment process, where the possibility of attachement is distinctly not independent of prior conditions. When a series of independent and identically distributed variables is multiplied together, the product's distribution converges on a log-normal distribution by the Central Limit Theorem.

Mitzenmacher argues that subtle variations in generative processes can turn their results one way or another. Ideally one can look more deeply at the structure of data, not just its distribution, to determine the process behind a heavy-tailed data set. Despite this difficulty, the statistical consequences of different processes will become more apparent asymptotically as more data is generated.

In summary, a process of network growth according to which degree is the result of a independent multiplicative process will assymptotically produce a log-normal distribution. A process of network growth driven by non-independent preferential attachment will approach a power law degree distribution. A test of the $\log$ likelihood of the best fit of either distribution on an empirical data set provides empirical support for the data's being produced by one process or the other.

\section{Degree assortativity}

Another graph theory concept that we will use in our analysis of collaborative mailing lists is degree assortativity. Degree assortativity is the correlation between degrees of adjacent nodes in the network. In the context of social networks, it is a measure of a special case of homophily, the tendency of people to be connected to others who are similar to them. Degree assortativity means that the most connected members of the network are connected with each other.

Following the mathematical definition of [Newman2003], the degree assortativity coefficient is

$$
r=\frac{\left.\sum_{j k} j k\left(e_{j k}-q_{j} q_{k}\right)\right)}{\sigma_{q}^{2}}
$$

In the above formula, $e_{j k}$ is the fraction of edges that connect vertices of degree $j+1$ and $k+1$, i.e. the degrees of the connected vertices not including the connecting edge itself. [Newman2003] calls this excess degree. The value $q_{k}$ is the distribution of excess degree.

$$
q_{k}=\sum_{j} e_{j k}
$$

The value $\sigma_{q}$ is the standard deviation of $q_{k}$.

[Newman2002] studied degree assortativity in complex networks and introduced an intriguing hypothesis. Observed social networks, such as those of academic coauthorship networks and business director associations, exhibit positive degree assortativity. Technical and biological networks, such as connections between autonomous systems on the Internet, protein interactions, and neural networks, exhibit negative degree assortativity, or disassortivity. Our own speculative interpretation is that the organization of technical and biological networks evolves for a functional purpose facilitated better by having highly connected hubs distributed widely, whereas many social networks are organized more according to the self-interest or homophilic tendencies of the participants.

[Noldus2015] reviews the extensive scholarship on assortativity in networks since Newman's work in 2002. They note that Barabási-Alpert networks are only slightly disassortive, converging on zero assortativity as the number of nodes increases. [Noldus2015] also surveys work such as [Newman2003] and [Foster2009] that define and analyze directed degree assortativity. In directed variations, degree assortativity is computed as above except using either the in-degree or out-degree of the source and targets nodes. In our empirical work below, we report directed assortativity in its in/in and out/out variations. We have observed little difference between these and the computed values for the in/out and out/in variations in our data, though there are theoretical graph structures for which these values can vary greatly.

According to the survey by [Noldus2015], assortivity in weighted networks is not well explored either theoretically or empirically. The weighted assortativity of a a network is the correlation between the weighted degree of its adjacent nodes, where weighted degree is the sum of the weights of all edges of a node. Directed weighted assortativity is computed from 
weighted in- and out-degrees. [Networkx] provides functions for computing these values on networks. We will compare weighted and unweighted directed assortivity in empirical networks below.

\section{Methods}

We collected archival data of 13 mailing lists from open collaborative communities. From these data we derived an interaction graph of who replied to whom. We then computed the weighted and unweighted degree assortativity of these networks. We also used the Alstott package to test the degree distribution of these networks using the Clauset method.

\section{Email data collection}

BigBang supports collection of email data. It can do this either by scraping the archival pages of a Mailman 2 instance, or by importing an .mbox formatted file. Internally, BigBang parses this data into a Pandas DataFrame [McKinney] and stores parsed and normalized email data in .csv format.

For the purpose of this study, we scraped data from public Mailman 2 instances associated with the following projects:

- SciPy: http://mail.scipy.org/mailman/listinfo/

- WikiMedia: http://lists.wikimedia.org/mailman/listinfo

- OpenStreetMap: http://lists.openstreetmap.org/listinfo

We selected mailing lists from the SciPy Mailman instance primarily for their relevance to the SciPy community. We also selected some mailing lists from other projects for comparison.

A limiting factor for our analysis is that every new data set introduces new edges cases BigBang's processing logic must take into account. For example, misformatted timestamps cause errors in many archival email data sets. In future work we hope to sample data more systematically in order to establish general principles of collaboration. This preliminery study is merely descriptive.

\section{Deriving interaction graphs}

Email is archived in the same text format that email is sent in, as specified in RFC2822 [RFC2822]. Every email is comprised of a message body and a number of metadata headers that are essential for email processing and presentation.

For our study, we have been interested in extracting the implied social network from an email archive of a public mailing list. To construct this network, we have used the From, Message-ID, and In-Reply-To headers of the email.

The From field of an email contains data identifying the mailbox of the message author. This often includes both a full name and an email address of the sender. As this is set by the email client and a single person may use many different mailboxes, a single person is often represented differently in the From field across many emails. See Entity Resolution for our strategies for resolving entities from divergent email headers.

The Message-ID header provides a globally unique identification string for every email. The uniqueness of the identifier must be guaranteed by the host that generates the message. It is recommended in [RFC2822] that email hosts accomplish this by including their domain name and combination of the exact date and time, as well as some other unique identifier (such as a process ID number) from the host system. The In-Reply-To header is set when an email is sent as a reply to another email. The reply's In-Reply-To header will match the Message-ID of the original email.
Formally, we construct the directed interaction graph $G$ from a set of emails indexed by $i \in I$. Each email consists of a tuple $\left(f_{i}, r_{i}\right)$, where $f_{i}$ identifies the mailbox of the sender (corresponding to the From header) and $r_{i} \in I \cup\{\varepsilon\}$ (corresponding to the In-Reply-To header) may be a null value $\varepsilon$ or be the index of another email.

- For every email $i$, if there is not one already add a node with label $f_{i}$ to $G$ corresponding and set its sent attribute 1. If such a node already exists, increment its sent attribute by 1 .

- Iterating again through every email $i$, if $r_{i} \neq \varepsilon$, and if there is not one already, then create a directed edge between nodes $f_{i}$ and $f_{r_{i}}$ with a weight attribute set to 1 . If the edge already exists, increment the weight attribute by 1 .

In sum, the final graph $G$ has a node for every email author annotated by the number of emails from that sender in the data set. There is an edge from $f_{i}$ to $f_{j}$ if author $f_{i}$ ever wrote a reply to an email authored by $f_{j}$. The weight of an edge corresponds to the number of these replies in the data set.

The motivation for constructing interaction graphs in this way is to build a concise representation of the social network implied by email data. We posit that building a social network representation based on actual messages sent between people provides a more granular and faithful description of social relationships than one based on higher-level descriptions of social relationships or ties from web services such as Facebook 'friends' and Twitter 'followers'

BigBang implements this interaction graph creation using Python's native email processing libraries, pandas, and networkx. [Networkx] The following code builds the interaction graph representations.

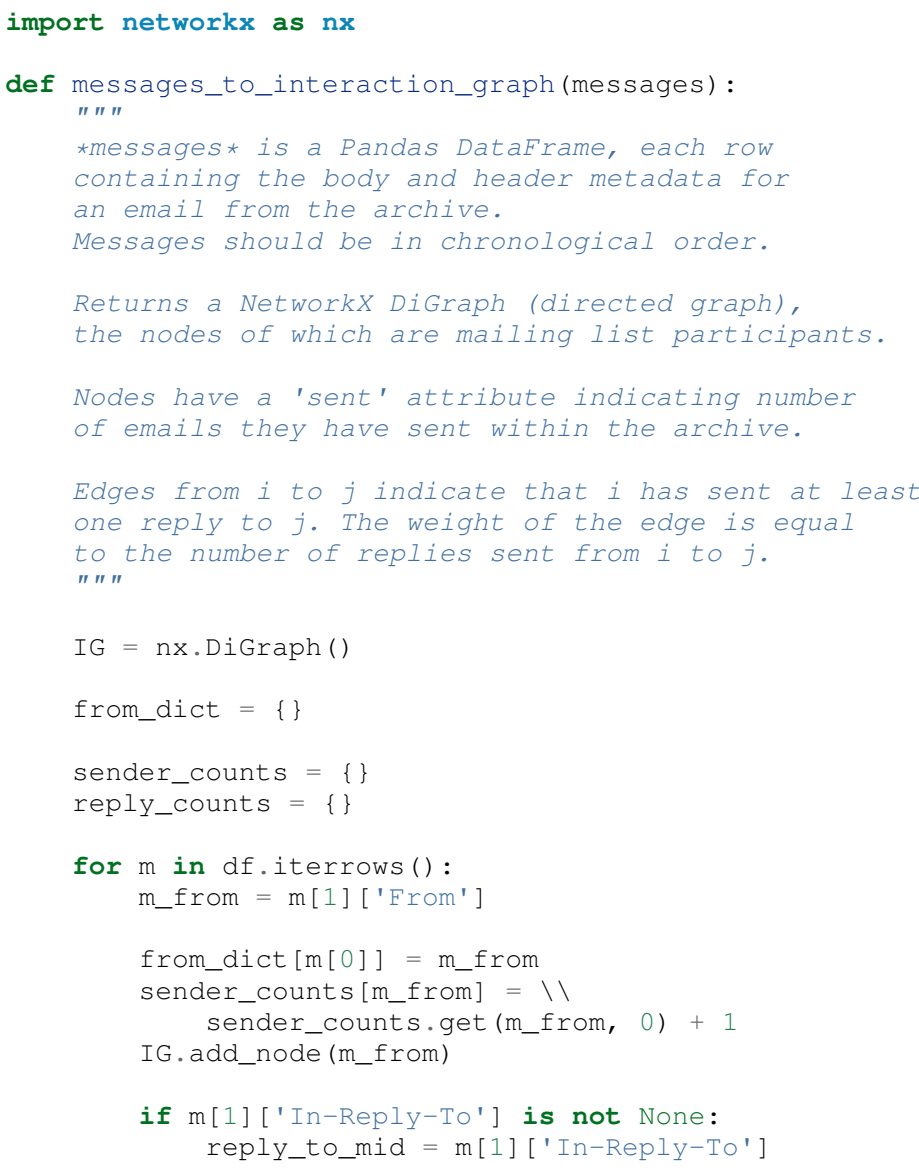




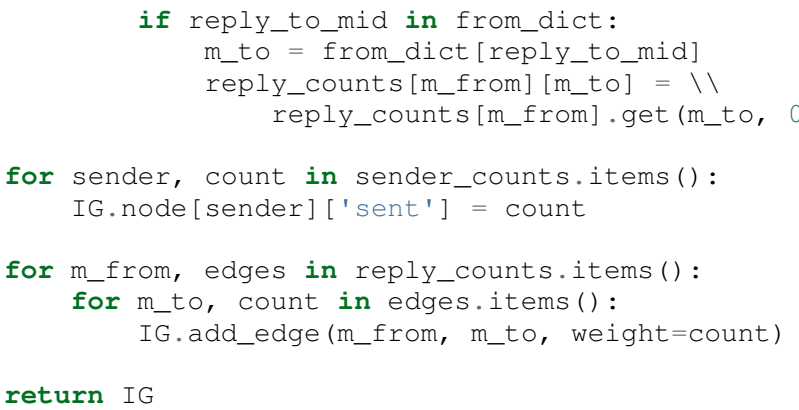

One potential objection to this approach is that since the data we are considering comes from public mailing lists where every message has a potentially large audience, it may be misleading to build a network representation on the assumption that a reply is directed primarily at the person who was replied to and not more broadly to other participants in a thread or, even more broadly, to the mailing list as a whole. While this is a valid objection, it points to the heart of what is distinctive about this research. While there have been many studies of social network formation in conventional settings, the conditions of open collaboration are potentially quite different. Theoretically, we expect them to be explicitly and collectively goal-directed, self-organized for efficient action as opposed to positional social advantage, and designed around an archiving system for the sake of efficiency. Understanding the statistical properties of this particular form of social organization, as opposed to others, is the very purpose of this empirical work.

\section{Entity Resolution}

Empirically, over the extent of a mailing list's archival data it is common for the From fields of emails to vary even when the email is coming from the same person. Not only do people sometimes change their email address or use multiple addresses to interact with the same list, but also different email clients may represent the same email address in the From header in different ways. BigBang includes automated techniques for resolving these entities, cleaning the data for downstream processing.

Data from the From header of messages stored by Mailman is most often represented in a form that includes both a full name representation and an email representation. Unfortunately these fields can vary widely for one person. Table 1 shows some of the variability that might appear for a single prolific sender. Variation in entity representation is a source of noise in our research and an ongoing area of development for BigBang.

For the study in this paper, we have implemented a heuristic system for entity matching.

- First we standardize the data by converting it to lower case and normalizing " at " and "@".

- Then we construct a similarity matrix between each entry. Each entry is parsed into email and full name subfields. The value of the similarity matrix at cell $(i, j)$ is 1 if there is an exact match of either the email address or the full name, and 0 otherwise.

- We then construct a graph from the similarity matrix and treat each connected component (group of nodes that are connected to each other by at least one path) as an entity.

Under this procedure, all of the above email addresses would be collapsed into a single entity. These heuristics were developed

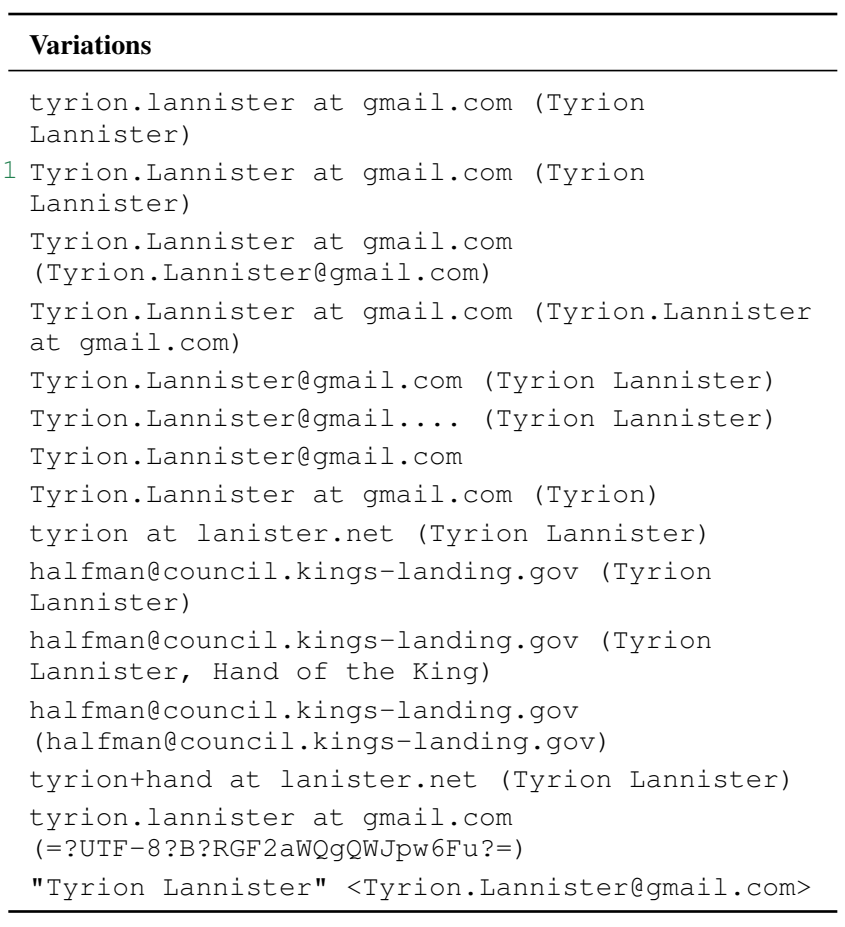

TABLE 1: Examples of variations in From header values corresponding to the same person in an email archive. Some of these changes reflect changes of email address. Others are artifacts of the users' email clients and the mailing list software.

through informal but thorough investigation of mailing list data we have analyzed for this paper. We leave it to future work to formally test and improve this method with respect to a sufficiently large and labeled test data set.

In our interaction graph study, this has the effect of combining several nodes into a single one in a way that's similar to the blockmodel technique. The edges to and from the derived node are weighted by the sum of the edges of the original nodes. The sent attribute of the new node is also set as the sum of the sent attribute of the original nodes.

\section{Results}

We computed the (unweighted) degre distribution and the weighted and unweighted degree assortativities of each of the mailing lists for which we collected data. We also aggregated the interaction graphs of each list into a single graph that we have called total and ran the same analysis.

Every mailing list of the 13 we analyzed exhibits degree disassortivity better fit to log-normal instead of power law distribution. This is the meaning of the negative $\mathrm{R}$ value given in Table 2 .

The $p$ values require special explanation. The value given is computed by the [Alstott2014] package in the direction of the best fitting distribution. Since the best fitting distribution is log-normal, the null hypothesis used for computing the $p$-values is that the data was generated from a power law distribution. That the $p$-value for no individual mailing list is beneath a threshold of statistical significance (such as $p<.05$ speaks to the similarity between these two distributions that is the source of such confusion and debate, as outlined previously. Especially for lists with low $n$, the [Clauset2007] test can be entirely inconclusive as to which distribution is more likely. 


\begin{tabular}{|c|c|c|c|c|c|c|c|c|}
\hline List name & List Source & $n$ & $R$ value & $p$ & in, in,weighted & out,out,weighted & in,in,unweighted & out,out,unweighted \\
\hline total & All sources & 9576 & -7.62 & 0.01 & -0.13 & -0.12 & -0.21 & -0.17 \\
\hline numpy-discussion & SciPy & 2973 & -0.76 & 0.40 & -0.22 & -0.20 & -0.29 & -0.26 \\
\hline scipy-user & SciPy & 2735 & -0.02 & 0.31 & -0.11 & -0.11 & -0.19 & -0.18 \\
\hline wikimedia-1 & WikiMedia & 1729 & -3.65 & 0.07 & -0.15 & -0.15 & -0.21 & -0.20 \\
\hline ipython-user & SciPy & 1085 & -0.33 & 0.23 & -0.27 & -0.26 & -0.29 & -0.26 \\
\hline scipy-dev & SciPy & 1056 & -0.33 & 0.58 & -0.28 & -0.26 & -0.31 & -0.29 \\
\hline ipython-dev & SciPy & 689 & -0.52 & 0.08 & -0.25 & -0.24 & -0.36 & -0.36 \\
\hline hot & OpenStreetMap & 524 & -0.85 & 0.40 & -0.19 & -0.20 & -0.24 & -0.24 \\
\hline astropy & SciPy & 404 & -0.08 & 0.77 & -0.16 & -0.20 & -0.16 & -0.16 \\
\hline gendergap & WikiMedia & 301 & -0.86 & 0.40 & -0.15 & -0.18 & -0.20 & -0.21 \\
\hline apug & SciPy & 121 & -0.01 & 0.52 & -0.20 & -0.20 & -0.21 & -0.22 \\
\hline maps-1 & WikiMedia & 118 & -0.00 & 0.95 & -0.19 & -0.18 & -0.27 & -0.26 \\
\hline design & WikiMedia & 111 & -3.62 & 0.10 & -0.18 & -0.17 & -0.21 & -0.21 \\
\hline potlatch-dev & OpenStreetMap & 75 & -0.00 & 0.97 & -0.01 & -0.08 & -0.45 & -0.34 \\
\hline
\end{tabular}

TABLE 2: Results of analysis. For each mailing list archive, number of participants $n$, loglikelihood ratio $R$ and statistical significance $p$ in the direction of the best fit. In all cases, the log-normal distribution is a better fit, though only in the case of the aggregated graph is the power-law distribution ruled out with statistical significance. We compute weighted and unweighted variations of (in,in) and (out,out) degree assorativity.

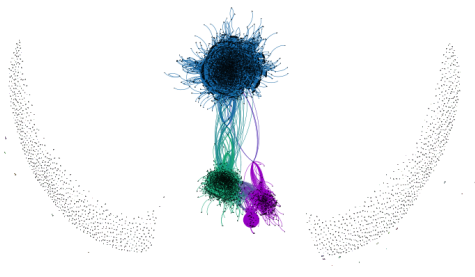

Fig. 1: Interaction graph of all participants across all mailing lists explored in this study, rendered with [Gephi]. The large blue module is roughly the SciPy community. The green module is the Wikimedia community. The purple module is the OpenStreetMap community. Notably, these communities are not completely disjoint. There are several bridge nodes, meaning there are some mailboxes that have participated in two or more of the communities represented. Singleton points on either side of the central component indicate email authors to whom nobody ever replied.

In the total interaction graph, we can rule out that the data was generated from a power law distribution because $p<.05$. One reason for this may be simply because the graph size $n$ is much larger than for any of the individual graphs. Another may be because of the sampling method of aggregating otherwise mainly separate networks.

We find in all cases that interaction graphs are disassortative. We have presented here the results of computing both weighted and unweighted variations of directed (in, in) and (out, out) assortativity. (In all cases, (out, in) and (out, in) were similar enough to the values given that we felt they provided no additional insight to the reader). We observe that the disassortativity of the interaction graphs appears to be insensitive to graph size $n$. We tentatively conclude that this disassortativity is therefore not of the residual sort found in small Barabási-Alpert graphs. A more thorough analysis of this point may be the subject of future work.

In most (but not all) cases, unweighted disassortativity in interaction graphs is more extreme than its weighted variation. As little work has been done on weighted degree disassortativity, we find this notable.

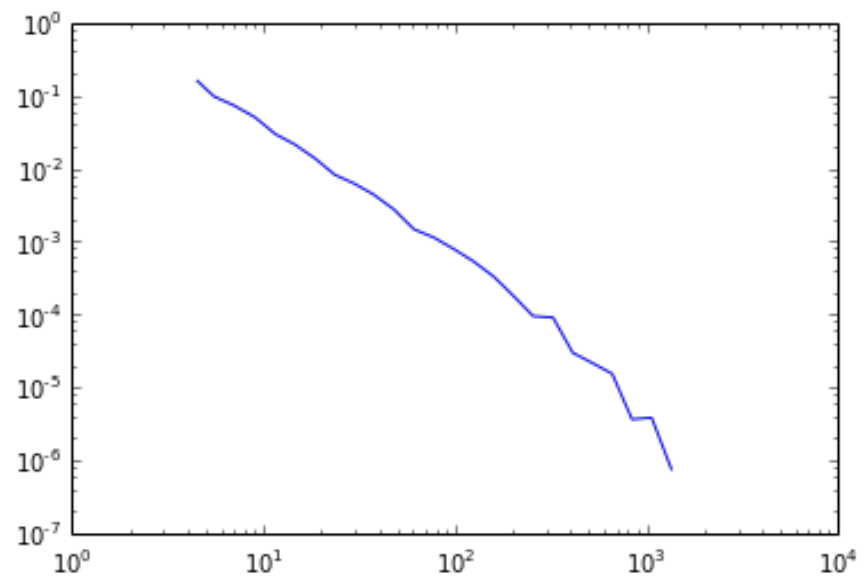

Fig. 2: A common technique for testing whether a distribution fits a log-normal or power law distribution is to plot its density function on log-log axes and observe whether the tail of the distribution drops below the line of best fit. As an illustration, this is the log-log plot of the probability density function for the unweighted degrees of the aggregated total interaction graph. In this paper, we eschew this technique on the grounds that it is biased for reasons discussed in depth in [Clauset2007].

\section{Discussion}

We have found no empirical support for email interaction networks having power law degree distribution, as opposed to a log-normal degree distribution. Interpretation of this result will vary depending on the "prior" probability on assigns to finding power law and log-normal distributions in social processes like this. As similar processes may generate both kinds of heavy-tail distributions, we can say only that our study suggests we should not be tethered to models that guarantee scale-free distributions such as BarabásiAlpert when explaining the interaction network data. We consider the development of a network generation model whose degree is determined by a multiplicative process as a direction for future work.

The statistical strength of the rejection of the power law 
hypothesis in the case of the aggregate interaction graph is noteworthy because it suggests that other social network analysis may suffer from a kind of myopia. Recall that preferential attachment requires that new nodes attach according to a probability distribution that is a global property of the network. But considering the growth of largely disjoint communities of collaborators, it is prima facie for one participant to understand the aggregate network structure. A network formation process that is more sensitive to this modularity may be a better fit for aggregated collaboration data.

A possible explanation for the disassortativity of these interaction graphs is a community norm of inclusiveness. If community leaders (who have high degree) make it a common practice to respond to new or infrequent participants in an effort to encourage them to contribute further, that would lead to disassortative mixing of degree. On the other hand, this mixing pattern could be the result of a much more generic statistical process.

It is our good fortune that the network data we study is granularly time-stamped. Since the total network structure is derived from an archive in which every email is annotated with a particular time, we see an opportunity to test generative models for there predictions during the whole duration of network growth.

Though anecdotally there is a difference between typical behavior on an open source project's developer list (e.g. ipythondev and scipy-dev in our data set) and a projects user list (e.g. ipython-user and scipy-user), these behavior differences do not surface as a clear statistical pattern in our study. A direction for future work is to more carefully operationalize and test for these behavioral differences.

We anticipate that research supported by BigBang will contribute to discourse on social roles in on-line communities [SocWik], [SocRole], measurement of digital labor [LaborWik], and the relationship between social structure and technical modularity [Zanetti2012].

We have also built BigBang and conducted this preliminary analysis with a number of applications in mind. One is anomaly detection in the open source ecosystem as a method of supply chain risk management. An statistical understanding of the typical patterns of collaborative behavior in open source software development could form the foundation for techniques that detect deviations from those patterns. If non-adherence to these patterns were correlated with propensity for software to be buggy or brittle, then detecting non-adherence could play a useful role in community self-management.

Another potential application of this research is in the appropriate incentivization of participation in open source development. Supposing, as seems likely, that open source software development is truly a collective effort and not merely the sum of many individual efforts, the question of how to best incentivize contributions to open source software is not an easy one. An understanding of how the network structure of collaboration relates to collective productivity could inform incentive plans that are sensitive to participants unique role within the network.

I gratefully acknowledge the helpful comments of Christine Choirat, Allen Downey, Thomas Kluyver, and Skipper Seabold.

\section{REFERENCES}

[Alstott2014]

Alstott J, Bullmore E, Plenz D (2014) powerlaw: A Python Package for Analysis of HeavyTailed Distributions. PLoS ONE 9(1): e85777. doi:10.1371/journal.pone.0085777
[AlbertBarabási]

Reka Albert and Albert-László Barabási. 2002 Statistical mechanics of complex networks. Reviews of Modern Physics, vol 74

[BarabásiAlbert] Albert-László Barabási \& Reka Albert. Emergence of Scaling in Random Networks, Science, Vol 286, Issue 5439, 15 October 1999, pages 509-512.

[Benthal12013] Benthall, S. 2013. "Reflexive Data Science: An Overview". http://dlab.berkeley.edu/blog/reflexive-datascience-overview

[Clauset2007] A. Clauset, C.R. Shalizi, and M.E.J. Newman. Powerlaw distributions in empirical data. arXiv:0706.1062, June 2007.

[Fogel] Fogel, K. 2013 Producing Open Source Software. http: //producingoss.com/

[Foster2009] Foster, J, Foster, D, Grassberger, P, and Paczuski, M. 2010 "Edge direction and the structure of networks" PNAS 2010107 (24) 10815-10820; published ahead of print May 26, 2010, doi:10.1073/pnas.0912671107

[Gephi] Bastian M., Heymann S., Jacomy M. (2009). Gephi: an open source software for exploring and manipulating networks. International AAAI Conference on Weblogs and Social Media.

[LaborWik] R. Stuart Geiger and Aaron Halfaker. 2013. Using edit sessions to measure participation in wikipedia. In Proceedings of the 2013 conference on Computer supported cooperative work (CSCW '13). ACM, New York, NY, USA, 861-870.

[McKinney] Wes McKinney. Data Structures for Statistical Computing in Python, Proceedings of the 9th Python in Science Conference, 51-56 (2010)

[Mitzenmacher2003] Mitzenmacher, M. 2003. "A Brief History of Generative Models for Power Law and Lognormal Distributions." Internet Mathematics Vol. 1, No. 2: 226-251

[Networkx] Aric A. Hagberg, Daniel A. Schult and Pieter J. Swart, "Exploring network structure, dynamics, and function using NetworkX", in Proceedings of the 7th Python in Science Conference (SciPy2008), Gäel Varoquaux, Travis Vaught, and Jarrod Millman (Eds), (Pasadena, CA USA), pp. 11-15, Aug 2008

[Newman2002] Newman, M. E. J. 2002. "Assortative mixing in networks."

[Newman2003] Newman, M. E. J. 2003. "Mixing patterns in networks." Phys. Rev. E 67, 026126

[Noldus2015] Noldus, R and Mieghem, P. 2015. "Assortativity in Complex Networks" Journal of Complex Networks. doi: 10.1093/comnet/cnv005

[RFC2822] Resnick, P. 2001. "Internet Message Format". Network Working Group, IETF.

[SocWik] Howard T. Welser, Dan Cosley, Gueorgi Kossinets, Austin Lin, Fedor Dokshin, Geri Gay, and Marc Smith. 2011. Finding social roles in Wikipedia. In Proceedings of the 2011 iConference (iConference '11). ACM, New York, NY, USA, 122-129.

[SocRole] Gleave, E.; Welser, H.T.; Lento, T.M.; Smith, M.A., "A Conceptual and Operational Definition of 'Social Role' in Online Community," System Sciences, 2009. HICSS '09. 42nd Hawaii International Conference on , vol., no., pp.1,11, 5-8 Jan. 2009

[Tinatti2012] Tinati, R., Carr, L., Hall, W. and Bentwood, J. (2012) Scale Free: Twitter's Retweet Network Structure. At Network Science 2012, Evanston, US.

[Zanetti2012] Zanetti, M. and Schweitzer, F. 2012. "A Network Perspective on Software Modularity" ARCS Workshops 2012, pp. 175-186.

[Zhou2011] Zhou T, Medo M, Cimini G, Zhang Z-K, Zhang Y-C (2011) Emergence of Scale-Free Leadership Structure in Social Recommender Systems. PLoS ONE 6(7): e20648. 